\title{
A note from the editors
}

\author{
Geoffrey S Ginsburg*,1, Jeanette J McCarthy ${ }^{1}$ \& George P Patrinos ${ }^{2}$ \\ ${ }^{1}$ Duke Center for Applied Genomics \& Precision Medicine, Duke University, Durham, NC USA \\ ${ }^{2}$ University of Patras, Patra, Greece \\ *Author for correspondence: Geoffrey.Ginsburg@Duke.edu
}
"We believe that the field of personalized and precision medicine is poised for impact, yet uptake into clinical practice faces significant hurdles. Therefore, the journal will focus on the path to translation and impact of those discoveries"

First draft submitted: 3 May 2018; Accepted for publication: 9 May 2018; Published online: 21 June 2018

Personalized Medicine, the journal, is entering a new era. With a new senior editorial team and support of the publisher, we are 'relaunching' Personalized Medicine and will be giving it new editorial priorities and content. Since the journal's original launch in 2004, the field of personalized medicine has undergone significant transitions. The human-genome project completed in 2003 gave rise to the notion that having access to human-genome sequence information alone will radically change medicine. That, it turns out, was naive in its simplicity. We now appreciate more than ever the complex interaction between genes, lifestyle, and environment. This in turn has led to the increasing use of digital technologies (wearables, mobile health and the extraordinary growth of data stored in electronic medical records) to track behaviors and collect biometric data, and the requisite growth of sophisticated data analysis including artificial intelligence, as a means to continuously monitor people and shed light on how these phenotypic traits are associated with genomics. The related term 'precision medicine' may capture this more holistic view and, in the journal, we wish to embrace the changes in the field and publish both precision and personalized medicine content. We recognize that convergence of genome-wide technologies with digital and data science, and the importance of individuals and their preferences is what is enabling significant changes in how we approach health, disease and health-to-disease transitions and their management.

Many significant meritorious scientific publications highlight the powerful tools of genomics in the discovery of novel underlying etiologies of disease. Others showcase the use of novel predictive molecular and clinical models for disease susceptibility, prognosis and responses to therapy. We believe that the field of personalized and precision medicine is poised for impact, yet uptake into clinical practice faces significant hurdles. Therefore, the journal will focus on the path to translation and impact of those discoveries and will report on fields that are less well-represented in the personalized medicine literature, implementation science and outcomes research as well as precision public health. In addition to encouraging investigators to submit papers along these lines, we will focus on work highlighting key strategies and data/research for evidence generation, participant engagement, data science, economic evaluation and health technology assessment of new genomic and related technologies, and training the next generation of clinicians and scientists. Primary research as well as longer review-type articles and shorter opinion pieces will all be encouraged and welcomed.

We also recognize the critical nature of a policy agenda to enable the field to advance, particularly as far as pricing and reimbursement as well as regulatory approval of precision medicine interventions are concerned. We wish to encourage analysis of issues on privacy, security, data sharing, regulations and reimbursement. We hope to have policy featured in all issues of the journal given its importance to forging a path for the science and clinical applications.

Diversity in genomics and personalized medicine research remains a key unmet scientific and clinical need. Minorities are underrepresented in most areas of clinical genomic research. Consequently, molecular diagnostic tests may not be generalizable across ethnic groups. In addition, there remain economic barriers to accessing personalized medicine by all. We hope to see future submissions along these lines to fill the gaps and to create a more level-playing field for personalized medicine in practice. 
Personalized medicine is a global agenda. Many nations are facing similar challenges and might be better positioned to have impact through collaboration. We look forward to the journal working closely with the Global Genomic Medicine Collaborative (G2MC, https://g2mc.org/) as the goals of the journal and the G2MC are very much aligned. G2MC is a nascent organization seeking to foster collaborative frameworks and knowledge dissemination on the implementation of genomic and personalized medicine around the world.

Finally, we are most pleased with the recent listing of Personalized Medicine in the Medline. This is an important milestone of the journal and one that signals continued growth and success. We invite all of you to contribute and to help take Personalized Medicine to its next level of impact on medicine and society.

\section{Financial \& competing interests disclosure}

The authors have no relevant affiliations or financial involvement with any organization or entity with a financial interest in or financial conflict with the subject matter or materials discussed in the manuscript. This includes employment, consultancies, honoraria, stock ownership or options, expert testimony, grants or patents received or pending, or royalties.

No writing assistance was utilized in the production of this manuscript. 